\title{
Monitoring after radiofrequency ablation of liver tumors: contrast-enhanced ultrasound vs. contrast-enhanced computer tomography, two days after procedure
}

\author{
Eva Korcakova ${ }^{\mathrm{a}, \mathrm{c}}$, Hynek Mirka ${ }^{\mathrm{a}, \mathrm{c}}$, Vaclav Liska ${ }^{\mathrm{b}, \mathrm{c}}$, Petr Hosek ${ }^{\mathrm{c}}$, Kristyna Bajcurova ${ }^{\mathrm{a}, \mathrm{c}}$
}

\begin{abstract}
Aim. Our research evaluated the ability of contrast-enhanced ultrasound (CEUS) and contrast enhanced computer tomography (CECT), performed $48 \mathrm{~h}$ after radiofrequency ablation (RFA), to detect residual tumor tissue. As recently published studies have evaluated periprocedural CEUS performed within $24 \mathrm{~h}$ after procedure and their results were not satisfactory, it seems that postponing the control test by one day could increase the sensitivity of both methods. Patients and Methods. We evaluated 33 patients with 37 lesions, who met the criteria for our study. The criteria were 1) the treated lesion is sonographically viewable and 2) the patient underwent both CEUS and CECT control $48 \mathrm{~h}$ after treatment and additional follow-up 3 months later by CECT. Presence of residual tumor tissue and the size of necrosis were recorded and compared to the results of 3-months CECT.

Results. Nine residues were present in our group. CEUS and CECT showed similar sensitivity $(66.7 \%$ and $77.8 \%$, respectively) and identical specificity (both 96.4\%) for detection of residual tumor tissue. CEUS significantly underestimated the size of necrosis in comparison to CECT (on average by $2.4 \mathrm{~mm}, P=0.0005$ ). Over the 3-months follow-up period the size of necrosis decreased in all patients (on average by $7.2 \mathrm{~mm}, P<0.0001$ ).

Conclusion. The quality of tumor residue detection in 48-h CEUS was comparable to that of $48-h$ CECT. This finding suggests the CEUS being a feasible substitute for CECT. Both methods show reasonable sensitivity; therefore this timing seems to be appropriate for the first post-treatment control while also allowing for early retreatment if residual tumor tissue is found.
\end{abstract}

Key words: contrast-enhanced ultrasonography, radiofrequency ablation, liver tumors, residual tumor tissue

Received: November 25, 2017; Accepted with revision: May 9, 2018; Available online: May 23, 2018 https://doi.org/10.5507/bp.2018.025

${ }^{a}$ Department of Imaging Methods, Teaching Hospital in Pilsen and Faculty of Medicine in Pilsen, Charles University, Pilsen, Czech Republic ${ }^{b}$ Department of Surgery, Teaching Hospital in Pilsen and Faculty of Medicine in Pilsen, Charles University, Pilsen, Czech Republic 'Biomedical Center, Faculty of Medicine in Pilsen, Charles University, Pilsen, Czech Republic Corresponding author: Eva Korcakova, e-mail: korcakovae@fnplzen.cz

\section{INTRODUCTION}

Primary liver tumors are the 5 th most common tumor type in men and the 9th most common tumor type in women worldwide. The most common primary liver tumor is hepatocellular carcinoma (HCC). It occurs frequently in the Middle East Asia and Africa, where there is a high incidence of chronic hepatitis $\mathrm{B}$ and $\mathrm{C}$ and increased exposure to aflatoxins. Occurrence of $\mathrm{HCC}$ is also higher in southern Europe, due to higher incidence of hepatitis C. Metastatic liver involvement is also common, especially in cases of colorectal carcinoma (CRC) and breast carcinoma. The liver is the first site to which metastatic CRC spreads. Approximately $50-60 \%$ of patients with CRC develop liver metastases during their life. According to the World Health Organization (WHO) cancer report, in 2012 liver cancer was the second most common cause of cancer-related death worldwide ${ }^{1}$.

Current curative methods include surgery and liver transplantation. In patients with healthy liver parenchyma, up to $70 \%$ of the liver can be resected, whereas only $20 \%$ can be resected in patients with liver cirrhosis ${ }^{2}$. Although modern treatment offers a number of options in the form of repeated surgery and combined operations, there are many patients for whom surgery is inappropriate. Patients with primarily advanced tumors or with multiple metastases affecting both hepatic lobes, patients with a small capacity of functional liver parenchyma (Child Pugh score $\mathrm{B}$ and $\mathrm{C}$ ) and patients with comorbidities cannot undergo major hepatic surgery ${ }^{3}$. The limitation for liver transplantation is primarily the small number of suitable donors.

Alternatives for these patients are methods for local destruction of the tumor, which are divided into physical methods and embolization. Physical methods are radiofrequency ablation (RFA), microwave ablation and cryotherapy. Currently, the most commonly used method for embolization is transarterial chemoembolization (TACE). RFA is a suitable method for treating a non-resectable single tumor $\leq 5 \mathrm{~cm}$ in diameter or maximum 3 lesions $\leq$ $3 \mathrm{~cm}$ in diameter ${ }^{4}$. The principle of the action is heating the tissue, causing dehydration and evaporation of water and thereby destroying the tumor cells ${ }^{5}$. Heat is produced as an effect of alternating current around the tip of the electrode. The effect of liver tumor treatment with RFA is 
monitored by imaging methods to detect residual tumor tissue, local recurrence or possible new tumor deposits.

After RFA, ablative necrosis develops, which presents in imaging as an avascular area. The residual tissue manifests as an atypically enhanced nodular structure at the edge of the necrosis. Enhancement of the residue is similar to the original tumor. HCC has an early intensive arterial enhancement followed by a washout. Metastases commonly have an enhanced rim in the early arterial phase, while in the late arterial phase they are similar to surrounding liver parenchyma and have significant washout. Soon after the procedure, a symmetrical hem of hyperemia may occur around the necrosis. The hem is regular and can cause problems with evaluation of the residue ${ }^{6}$.

CECT is usually used for monitoring. CEUS has been used for post-RFA monitoring for the past decade ${ }^{7}$. The obvious advantage of CEUS compared with CECT is the absence of radiation and the low allergic potential of the contrast medium, which is eliminated from the body via the lungs and does not damage the kidneys and thyroid gland. Other advantages include the possibilities of realtime monitoring and repeating the examination with additional contrast doses, without damage to patient's health. Disadvantages include the subjectivity of CEUS, similar to other US examinations, and dependence on the investigator's experience. The primary problem is the unavailability of some lesions, especially deep-seated lesions in obese patients and after operation due to adhesions and the lower sensitivity for lesions in areas accessible with difficulties ${ }^{8}$.

\section{PATIENTS AND METHODS}

We evaluated 47 consecutive patients, treated between the years 2014 and 2016 for liver tumors with percutaneous RFA in our department. All patients agreed to undergo interventional procedures and pretreatment and post-treatment examinations. All patients gave written informed consent. The study was approved by the local ethics committee and was performed in accordance with the last revised version of the Helsinki Declaration.

The first CEUS examination was performed in the morning on the day of the procedure. The aim of this examination was to assess the size and location of the lesion and the type of enhancement. Accessibility of lesions at baseline examination was rated on 3-point scale: 1) easily accessible lesion and good visibility; 2) limited visibility, but still sufficient for evaluation (lesion visible transcostally depending on breathing movements, with little saturation); and 3) unavailable or invisible deposits, which are inappropriate for further investigation by CEUS.

The inclusion criteria for our study were as follows: 1) the treated lesion is sonographically viewable; 2) the patient had undergone both CEUS and CECT control 48 $\mathrm{h}$ after RFA, as well as an additional follow-up 3 months later; and 3) there is no generalization at the 3-months follow-up examination. The exclusion criteria were as follows: 1) impossibility to detect lesions on CEUS, 2) al- lergy to iodinated contrast medium, and 3) multiple new liver lesions identified with CECT.

In the initial cohort 6 patients had poor visibility of lesions and 4 patients had iodine allergies. Due to different follow-up schedules for magnetic resonance (MR) monitoring, we excluded these patients from subsequent evaluation. We also excluded 4 patients who had generalized multi-metastatic disease in the liver at the examination 3 months after RFA.

A total of 33 patients met the inclusion criteria (Table 1). Four patients had two treated lesions; for the purposes of this study we evaluated each lesion separately. The total number of evaluated lesions was thus 37 .

Patients underwent the CEUS and CECT $48 \mathrm{~h}$ after RFA. Post-procedural CEUS and CECT were blindly reviewed by two radiologists. They answered the following questions: 1) is any type of tumor tissue residue present? $(\mathrm{Y} / \mathrm{N}) ; 2)$ is there a symmetrical hem of enhancement around the lesion with indicates the periablastic edema? $(\mathrm{Y} / \mathrm{N}) ; 3)$ what is the size of the necrosis and does it overlap the original lesion site? Outcomes were compared with results of 3-months follow-up CECT examination, which were used as the reference value. The results were established by consensus.

CEUS was performed with Acuson SL 2000 (Siemens, Erlangen, Germany) or Aplio 500 (Toshiba, Tokyo,

Table 1. Characteristics of evaluated patients $(n=33)$.

\begin{tabular}{lc}
\hline Sex & \\
Male & $23(70 \%)$ \\
Female & $10(30 \%)$ \\
Age at the time of treatment (median, range) & $66(30-85)$ \\
Number of lesions per patient & \\
one & $29(89 \%)$ \\
two & $4(11 \%)$ \\
Size of the tumor before procedure $(\mathrm{n}=37)$ & \\
$1-15$ mm & $11(30 \%)$ \\
$16-30$ mm & $17(46 \%)$ \\
$31-50$ mm & $7(19 \%)$ \\
51 and more & $2(5 \%)$ \\
Histology & \\
HCC & $10(27 \%)$ \\
Metastases of colorectal carcinoma & $20(54 \%)$ \\
Metastases of breast carcinoma & $6(16 \%)$ \\
Other & $1(3 \%)$ \\
Location & \\
S1+2+3 & $19(51 \%)$ \\
S4 & \\
S5+6 & \\
Seasibility & $31(83 \%)$ \\
Great & $6(17 \%)$ \\
Medium & $9(24 \%)$ \\
\hline
\end{tabular}


Japan) sonographic scanner using contrast-specific software, which operates with a low mechanical index. Examinations were carried out by two trained radiologists, each with 20 years of experience in ultrasound diagnostics. The contrast medium in the amount $2.4 \mathrm{~mL}$ of sulphur hexafluoride solution (Sonovue, Bracco, Italy) per dose was flushed by $10 \mathrm{~mL}$ saline solution. Every evaluated lesion was monitored continuously for the first $90 \mathrm{~s}$ after intravenous application of the contrast medium. The procedure was recorded on video, which was stored on the Picture Archiving and Communication System (PACS). Subsequent observation took $5 \mathrm{~min}$.

CECT was performed on a Definition Flash dualsource multidetector scanner (Siemens, Erlangen, Germany) after administration of iodine-based contrast medium (300 mg Iodide per $\mathrm{mL}$, flow rate $4 \mathrm{~mL} / \mathrm{s}$, amount $60-100 \mathrm{~mL}$ depending on patient weight) with a flush of $100 \mathrm{~mL}$ saline. The abdomen was scanned in two phases, arterial phase (bolus tracking in descending aorta, density $100 \mathrm{HU}$, delay $5 \mathrm{~s}$ ) and portal phase (delay $40 \mathrm{~s}$ ).

Standard monitoring in our institution is being performed 48 h, 3 months, 6 months and 12 months after RFA and then at six-months intervals.

RFA procedures were performed percutaneously under conscious sedation induced by an anesthesiologist. An internally-cooled Cool-tip ablation system E series electrode (Covidien, Minneapolis, USA) was introduced under CT or ultrasound (US) guidance. Total time of treatment was $12 \mathrm{~min}$. If residue was suspected, the electrode was redirected and another cycle of ablation performed.

\section{Statistical analysis}

Detection of residual tumor tissue was evaluated in relation to the results of 3-months follow-up CECT that were considered the true condition. Standard diagnostic accuracy indicators (sensitivity, specificity, positive and negative predictive value) of 48-h CEUS and 48-h CECT were calculated and compared. Significance of their differences was assessed using Fisher's exact test. Accuracy of necrosis size measurement by CEUS was assessed by investigating the distribution of the differences between measurement results of 48-h CEUS and 48-h CECT (using Bland-Altman plot, Pearson's correlation, ShapiroWilk test, one-sample t-test and standard descriptive statistics for normal distribution). The change of necrosis size over the 3-month follow-up period was analyzed in the same way from the difference between the 48-h CECT and 3-months CECT. All reported p-values are two-tailed and the significance level was set at $\alpha=0.05$. The statistical analysis was performed using Statistica (version $12 \mathrm{Cz}$, TIBCO Software Inc., Palo Alto, CA, USA).

\section{RESULTS}

Nine tumor residues were detected at the 3-months follow-up CECT. $48 \mathrm{~h}$ after ablation both methods agreed upon 5 of these residues. In the remaining residues, CEUS produced three false negatives and one additional true positive, while CECT produced two false negatives and two additional true positives. Interestingly, only one false negative finding was reported by both methods for the same residue, we speculate that the residue was very small and at the first follow-up control invisible. The remaining false negatives were scattered across different lesions. CEUS and CECT both produced one false positive for which the second method reported a true negative.

Sensitivity of CEUS was 66.7\% (95\% CI 29.9-92.5\%), whereas specificity was $96.4 \%$ (81.7-99.9\%). Positive predictive value (PPV) of CEUS was $85.7 \%$ (45.3-97.8\%), whereas negative predictive value (NPV) was $90.0 \%$ (78.1-95.8\%). Sensitivity of CECT was $77.8 \%$ (95\% CI 40.0-97.2\%), whereas specificity was 96.4\% (81.7-99.9\%). PPV of CECT was $87.5 \%$ (49.8-98.0\%) and NPV was $93.1 \%(79.9-97.9 \%)$. None of the observed differences was statistically significant (all $P=1$ ).

The deviations of 48-h CEUS from 48-h CECT (i.e., CEUS measurement error) were distributed close-tonormally $(P=0.6950)$ with significantly non-zero mean
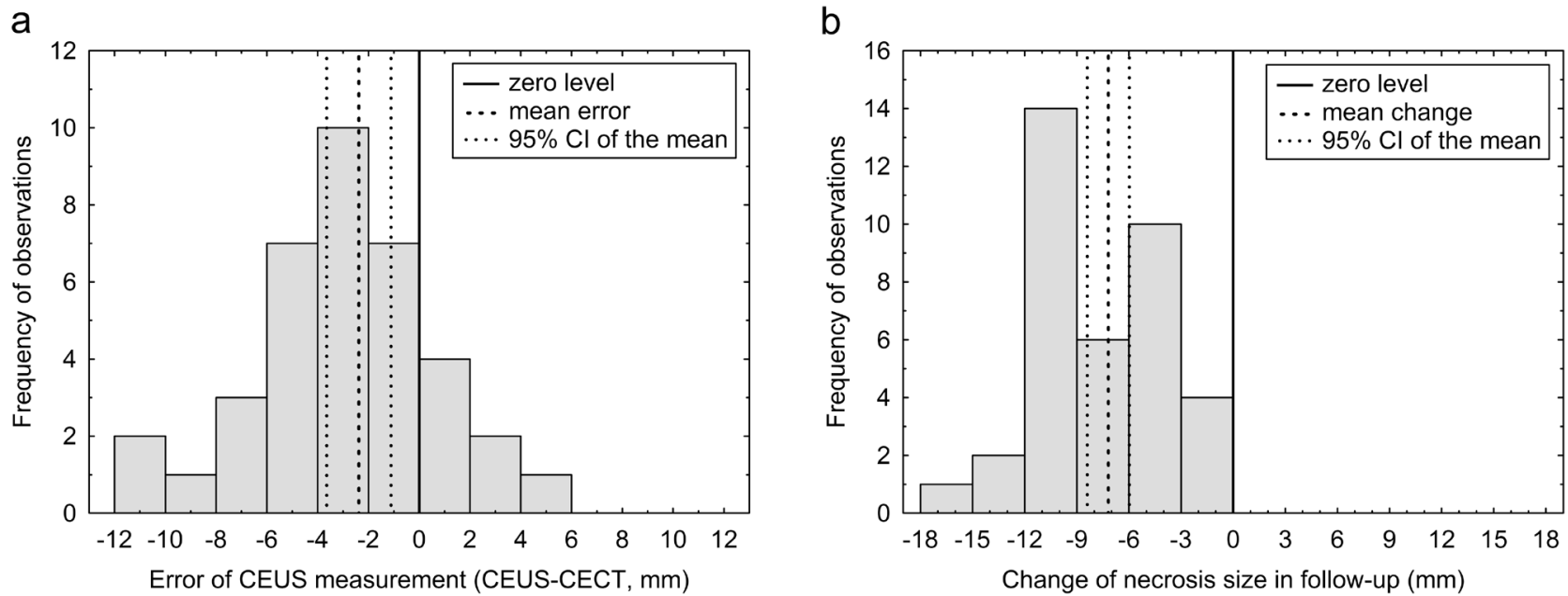

Fig. 1. Histograms of differences in necrosis size measurements between: (a) 48 h CEUS and 48 h CECT and (b) 48 h CECT and 3-months CECT. 
$(P=0.0005)$. The mean error was $-2.38 \mathrm{~mm}$ (95\% CI -3.64 - $-1.11 \mathrm{~mm})$, implying that the CEUS significantly underestimated the size of necrosis by an average of $2.38 \mathrm{~mm}$ (Fig. 1a). There were no signs of the CEUS's error being related to the size of the necrosis (Pearson's $r=0.019$; $P=0.911)$.

In comparison between the 48-h CECT and 3-months CECT, the size of necrosis decreased in all patients (mean change -7.19 mm with 95\% CI -8.40 - -5.98 mm; significantly non-zero with $P<0.0001)$. We found no association between the extent of necrosis' reduction and the initial size of the necrosis (Pearson's $\mathrm{r}=-0.301 ; P=0.070$ ) (Fig. 1).

\section{DISCUSSION}

CEUS is widely usable in patients with liver tumors treated by thermal ablation. CEUS can be used in primary diagnosis for specification of the tumor's enhancement and for precise pre-operative planning. In our opinion, pre-procedural CEUS is advantageous for assessing the accessibility of the lesion and its enhancement characteristics for further monitoring and for ruling out additional lesions. In particular, in case of hypovascular metastases that do not have intensive arterial enhancement, it is necessary to know the precise position of the original deposit. For complete treatment by ablation, it is also necessary to know the exact present extent of the tumor ${ }^{9}$.

CEUS can additionally be used to target the electrode under US control, especially for lesions with poor inherent visibility but good arterial supply ${ }^{10,11}$. Some operators use CEUS to monitor the immediate ablation effect approximately 5-10 min after ablation. In the periprocedural period CEUS is advantageous for assessing the effect of ablation ${ }^{11}$. Mauri et al. reports benefits of intra-procedural use of CEUS ( ref. $^{12}$ ). Immediate detection of residue allows immediate retreatment. The authors demonstrate a significant reduction in the number of residues, a decrease in the frequency of second ablation sessions, better patient comfort, and reductions in treatment cost. The study by Lekht showed better results in the group monitored intra-procedurally with CEUS than in the group without CEUS control ${ }^{13}$. We do not use intra-procedural CEUS to guide RFA.

At the post-procedural examination, we measured the size of the necrosis and compared its position with the position of the treated tumor. The recommended procedure for assessing the size of the necrosis includes measuring the longest dimension and the dimension perpendicular to it $^{14}$. A sufficient safety margin is considered to be $5 \mathrm{~mm}$ for HCC and $10 \mathrm{~mm}$ for metastasis. However, in practice, such a wide safety margin is not usually attained. Our results showed that CEUS indicated smaller size of necrosis compared to CECT, the mean difference was $2.4 \mathrm{~mm}$. This error was caused by the different measurement plane. CEUS does not have a standard measurement plane compared to CECT where we use the axial plane. A limitation of RFA can be the heat sink effect, which is caused by blood flow in nearby blood vessels and cooling of the surrounding tissue. When the heat sink effect is more pronounced, it can cause deformation of the necrosis. Irregular necrosis shapes occur in patients, with altered thermal conductivity in liver tissue caused by parenchymal damage due to systemic therapy.

First post-procedural CEUS was performed $48 \mathrm{~h}$ postRFA in our cohort, in contrast to most published studies. The reason for this timing is that early post-procedure CEUS could have lower sensitivity. Meloni demonstrated a significantly smaller ablation zone on intra-procedural CEUS compared to CEUS performed $24 \mathrm{~h}$ after ablation. The same study demonstrated $20 \%$ sensitivity for $24 \mathrm{~h}$ CEUS and $40 \%$ for $24 \mathrm{~h}$ CECT (ref. ${ }^{15}$ ). Similar results were reported in the study by Vilana, which observed $27 \%$ sensitivity for CEUS and 20\% sensitivity for CECT performed $24 \mathrm{~h}$ post procedure ${ }^{16}$. Wu et al. demonstrated in a rat liver model that the best time to evaluate the size of thermal coagulation caused by RFA is on the second day after treatment, when the necrosis is largest ${ }^{17}$. In our study the sensitivity of examination $48 \mathrm{~h}$ after ablation seemed higher compared to results reported $24 \mathrm{~h}$ postablation. Also, patients are still in the hospital at this time, therefore it is easy organize subsequent treatment if necessary. On the other hand, the rim of hyperemia around the ablation zone results in reduced evaluability. The rim can last for several weeks. CECT typically displays a more intensive rim of enhancement around the necrosis due to hyperdense contrast medium in the vessel and the movement of contrast medium into the extracellular space from damaged vessels ${ }^{18}$. The CEUS contrast agent has a true blood-pool distribution, and therefore only vessels are hyperechoic (Fig. 2,3).

The residue of the hypervascularized tumor, which is typically HCC, manifests in CEUS as a nodular intensively enhancement lesion in arterial phase with early washout or as a vessel or group of vessels filled with contrast medium in necrosis. The residuum of metastasis typically presents as a zone of early wash-out in the periphery of the necrosis without early intense enhancement. Secondary liver tumors have higher prevalence than primary tumors in our population, therefore it is very important for our work to know tumor enhancement characteristics prior to treatment, because metastases of various tumors behave in an unpredictable way (Fig. 4).

It is important to measure the size of post-ablative necrosis at regular controls. The size of the necrosis should gradually decrease as our results confirm. Between the 48-h CECT and 3-months CECT the necrosis was reduced by an average of $7 \mathrm{~mm}$. If there is no reduction in size of necrosis it means a suspicion of recurrence.

It seems to be sufficient to use CEUS separately for the first post-procedural examinations in patients without complications in connection with the interventions. CEUS should be supplemented with another crosssectional imaging method (CT, MR, or PET/CT) for long-term monitoring. CEUS is suitable for evaluation of treated lesions, but its sensitivity for detection of potential new lesions is unsatisfactory. The reason for this 


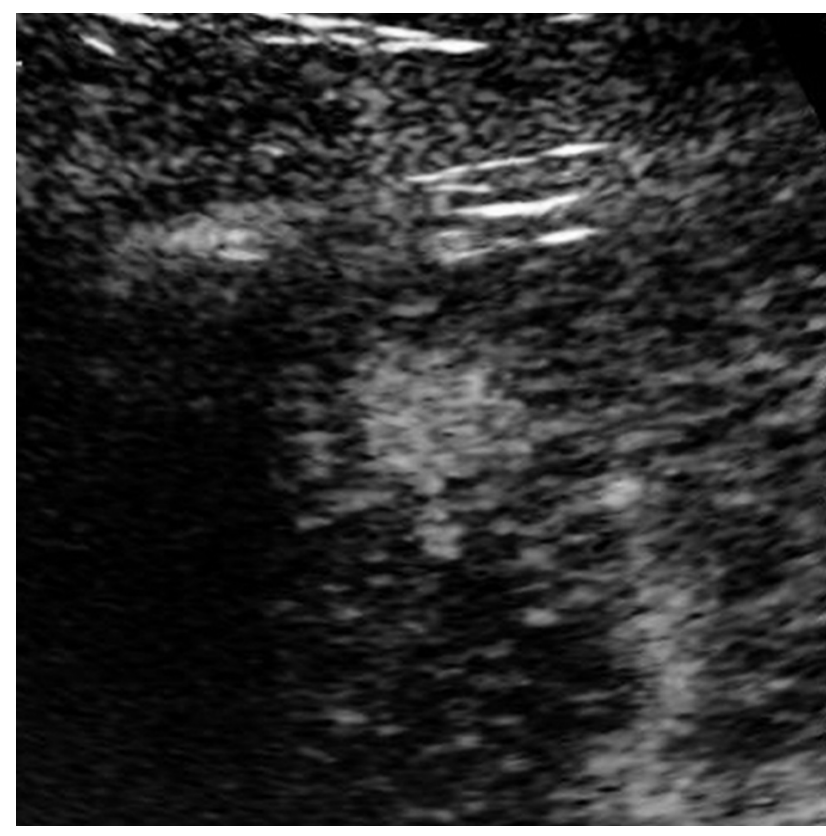

Fig. 2. Patient with HCC, CEUS examination in the morning before the procedure. Tumor with intensive enhancement in arterial phase.

low sensitivity could be the short duration of the arterial phase, which does not allow examination of the whole liver, particularly for tumors with high arterial enhancement $^{19}$. At our clinic, we combine CEUS with CECT for long-term monitoring after RFA.

Our study is limited by the small number of treated tumors and by the small amount of residues in our cohort. However, the infrequency of residues is a sign of good treatment by RFA. A second limitation is the predominance of metastases, which are generally less detectable.

\section{CONCLUSION}

CEUS is a suitable method for monitoring the treatment with percutaneous RFA. Multiple monitoring protocols have been published, but based on our experience, a 48-h post-procedural examination is the most advantageous timing. In our cohort, sensitivity for CEUS and CECT $48 \mathrm{~h}$ post-procedural was higher than published results from examination $24 \mathrm{~h}$ post-procedure; thus, a longer interval after the operation seems to be associated with better evaluation and better results. Based on our findings, CEUS is the most appopriate metod for the first control performed $48 \mathrm{~h}$ after treatment. For long term monitoring is CEUS ideally in combination with other cross-sectional imaging methods.

Acknowledgement: This study was supported by the National Sustainability Program I (NPU I) Nr. LO1503 provided by the Ministry of Education Youth and Sports of the Czech Republic and grant SVV 260392 provided by Charles University and by the project of the Ministry of Health of the Czech Republic - Conceptual Development of Research Institutions 00669806 - FN

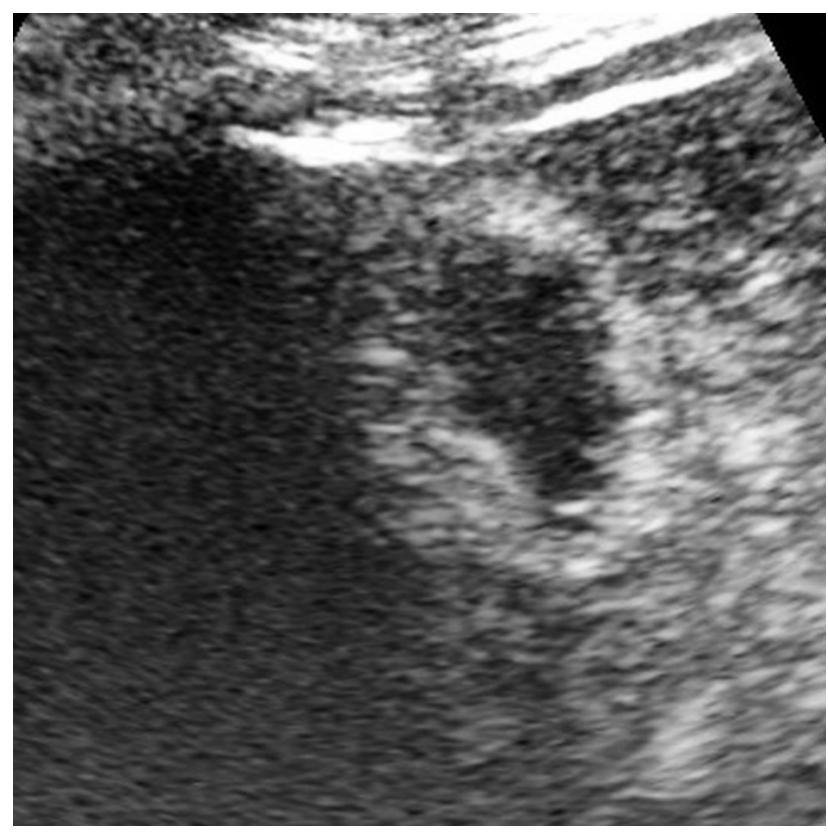

Fig. 3. CEUS $48 \mathrm{~h}$ after RFA, anechogenic necrosis covers the treated tumor. Regular rim of hyperemia around the ablation zone.

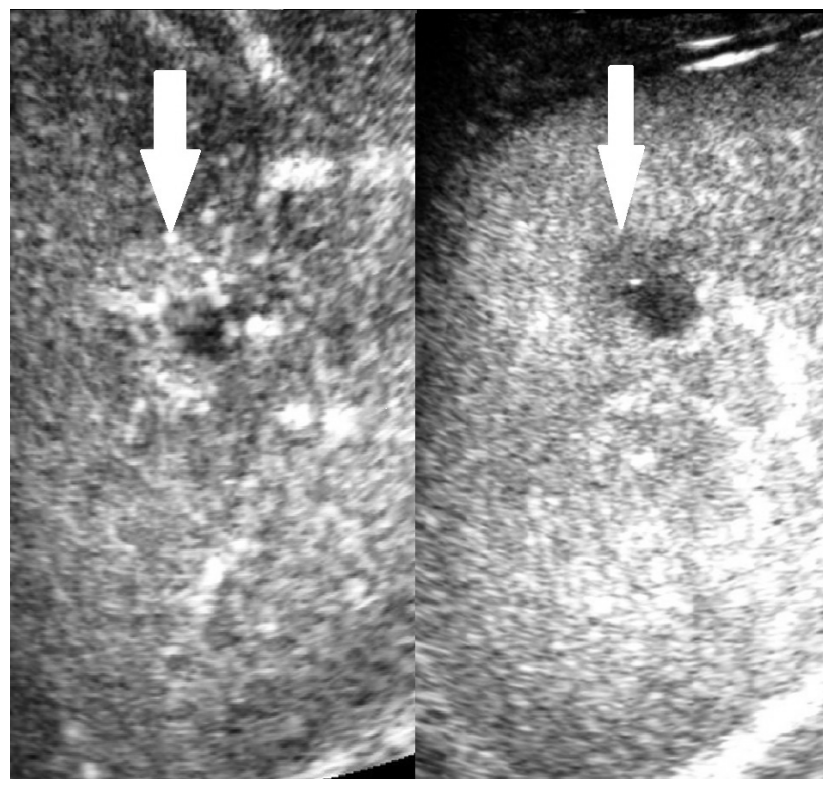

Fig. 4. Patient with HCC, 3 month after RFA, CEUS. Nodular hyperechoic area in arterial phase at the edge of necrosis and hypoechoic area in venous phase as the sign of early washout. It is a sign of recurence.

Plzen, by the project of the Charles University Prague Progress Q39, by the project "Centrum of clinical and experimental liver surgery" UNCE/MED/006 of the Charles University and by the project CZ.1.05/2.1.00/03.0076 from European Regional Development Fund.

Author contributions: All co-authors have read the final manuscript within their respective areas of expertise and participated sufficiently in the study to take responsibility for it and accept its conclusions.

Conflict of interest statement: None declared. 


\section{REFERENCES}

1. Stewart BW, Wild CHP. World cancer report 2014. Lyon: International agency for research on cancer; 2014. Chapter 5.6, Liver cancer; 403 412.

2. Skalický T, Třeška V, Šnajdauf J, Kala Z. Hepato-pankreato-biliárn chirurgie, Praha: Maxdorf 2011. Czech Republic

3. Bruix J, Shermann M. Management of hepatocellular carcinoma: An update. Hepatology 2011;53:1020-2.

4. Vogl TJ, Helmberger TK, Mack MG, Rieser MF. Percutaneous tumor ablation in medical radiology, Berlin: Springer-Verlag 2008.

5. Ahmed M, Brace CL, Lee FT, Goldberg SN. Principles of and advantaces in percutaneous ablation. Radiology 2011;258:351-66.

6. Sainani NI, Gervais DA, Mueller PR, Arellano RS. Imaging after percutaneous radiofrequency ablation of hepatic tumors: Part I, normal findings. AJR 2013;2001:184-93.

7. Claudon M, Dietrich CHF, Choi BI, Cosgrove DO, Kudo M, Nolsoe CHP Piscaglia F, Wilson SR, Barr RG, Chammas MC, Chaubal NG, Chen MH, Clevert DA, Correas JM, Ding H, Forsberg F, Fowlkes JB, Gibson RN Goldberg BB, Lassau N, Leen EL, Mattrey RF, Moriyasu F, Solbiati L, Weskott HP, Xu HX. Guidelines and good clinical practice recommendations for contrast enhanced ultrasound (CEUS) in the liver - update 2012 a WFUMB-EFSUMB initiative in cooperation with representatives of AFSUMB, AIUM, ASUM, FLAUS and ICUS. Ultrasound in Med \& Biol 2013;39:187-210.

8. Chung YE, Kim KW. Contrast-enhanced ultrasonography: advance and current status in abdominal imaging. Ultrasonography 2015;34:3-18.

9. Meloni MF, Smolock A, Cantisani V, Bezzi M, D'Ambrosio F. Contrast enhanced ultrasound in the evaluation and percutaneous treatment of hepatic and renal tumors. Eur J Radiol 2015;84:1666-74.

10. Sparchez Z, Radu P, Kacso G, Sparchez M, Zaharia T, Al Hajjar N Prospective comparison between real time contrast enhanced and conventional ultrasound guidance in percutaneous biopsies of liver tumors. Med Ultrason 2015;17(4):456-63.
11. Tondolo T, Mauri G, Solbiati L. Role and benefit of contrast-enhanced ultrasound during liver ablation procedures and follow-up. Solutions in contrast imaging 2012;12(3):1-7.

12. Mauri G, Porazzi E, Cova L, Restelli U, Tondolo T, Bonfanti M, Cerr A, lerace T, Croce D, Solbiati L. Intraprocedural contrast-enhanced ultrasound (CEUS) in liver percutaneous radiofrequency ablation: clinical impact and health technology assessment. Insights imaging 2014; 5:209-16

13. Lekht I, Gulati M, Nayyar M, Katz MD, Ter-Oganesyan R, Marx M, Cen SY, Grant E. Role of contrast-enhanced ultrasound (CEUS) in evaluation of thermal ablation zone. Abdom Radiol 2016;41:1511-21.

14. Meloni MF, Andreano A, Zimbaro F, Lava M, Lazzaroni S, Sironi S. Contrast enhanced ultrasound: Role in immediate post-procedural and 24-h evaluation of the effectiveness of thermal ablation of liver tumors. Journal of Ultrasound 2012;15:207-14.

15. Meloni MF, Andreano A, Franza E, Passamonti M, Lazzaroni S. Contrast enhanced ultrasound: Should it play a role in immediate evaluation of liver tumors following thermal ablation? European journal of Radiology 2012;81:e897-e902.

16. Vilana R, Bianchi L, Varela M, Nicolau C, Sánchez M, Ayuso C, García M, Sala M, Llovet JM, Bruix J, Bru C. Is microbubble-enhanced ultrasonography sufficient for assessment of response to percutaneous treatment in patients with early hepatocellular carcinoma? Eur Radiol 2006;16:2454-62.

17. Wu H., Wilkins LR, Ziats NP, Haaga JR, Exner AA. Real-time monitoring of radiofrequency ablation and postablation assessment: Accuracy of contrast-enhanced US in experimental rat liver model. Radiology 2014:270:107-16

18. Guibal A, Bertin C, Egels S, Savier E, Grenier PA, Lucidarme O. Contrast-enhanced ultrasound (CEUS) follow-up after radiofrequency ablation or cryoablation of focal liver lesions: treated-area patterns and their changes over time. Eur Radiol 2013;23:1392-400.

19. Roccarina D, Garcovich M, Ainora ME, Riccardi L, Pompili M, Gasbarrini A, Zocco MA. Usefulness of contrast enhanced ultrasound in monitoring therapeutic response after hepatocellular carcinoma treatment. World J Hepatol 2015;7(14):1866-74. 